\title{
THE NATURAL HISTORY OF THE RHEUMATOID METACARPO-PHALANGEAL JOINT
}

\author{
Michael McMaster, Oswestry, England \\ From the Rheumatism Foundation Hospital, Heinola, Finland
}

Rheumatoid arthritis is a systemic disease usually presenting as a chronic progressive symmetrical inflammation of multiple joints. The metacarpo-phalangeal joints are the commonest sites of involvement, the initial lesion being a proliferative synovitis. As the disease progresses the proximal phalanx commonly subluxates volarward and is deviated ulnarward on the metacarpal head (Smith and Kaplan 1967). The final outcome is complete destruction of the joint.

Radiographs have been used to study bone erosion in the metacarpo-phalangeal joints (Martel 1964; Martel, Hayes and Duff 1965; Norgaard 1965), but unfortunately these methods only reflect the gross bony pathology and give no indication of the early changes in the articular cartilage. It is important to understand the manner in which cartilage is altered because joint function is dependent on the integrity of its articular cartilage and this cartilage once destroyed does not regenerate.

The purpose of this study is threefold: firstly to observe the distribution of both bone erosion and cartilage degeneration in the rheumatoid metacarpo-phalangeal joint at all stages of the disease; secondly to investigate the factors governing the position and progression of these lesions; and finally to correlate the radiographic findings with the pathological changes in the joint.

\section{CLINICAL MATERIAL AND METHOD OF STUDY}

A prospective study was carried out on forty-five adult patients who were having operations on their metacarpo-phalangeal joints because of rheumatoid arthritis. The disease was considered either classical or definite in all patients according to the criteria of the American Rheumatism Association (1959).

There were thirty-seven females and eight males whose age distribution and duration of hand symptoms were as shown in Tables I and II. None of the metacarpo-phalangeal joints had received any intra-articular therapy.

Synovectomy of the metacarpo-phalangeal joints was performed on thirty-one patients, many of whom had minimal or no radiographic signs of bone erosion. This provided an opportunity to observe the very earliest changes within the joint. A Vainio type of metacarpophalangeal joint arthroplasty (Hellum and Vainio 1968) was performed on fourteen patients and in all of these patients the second to fifth metacarpal heads were resected although not all were involved to the same extent.

These two types of operation provided a total of 126 metacarpo-phalangeal joints for study at all stages of the disease. Sixty-seven joints were in left hands and fifty-nine in right. The distribution of the joints is as shown in Table III.

All of the joints were seen at operation by the author and a detailed diagram was made of the macroscopic appearance of each joint. The exact position and extent of the bone erosions, and the varying degrees of cartilage damage were noted and in many cases photographs were also taken.

The resected metacarpal heads were collected, and in order to show more clearly on photography the areas of normal cartilage from the areas of degenerate cartilage and bone erosion a differential staining technique was carried out. This involved briefly dipping the metacarpal heads in indian ink and forty-five seconds later washing them in running water. 
Normal cartilage remained unstained, degenerate pitted cartilage held some of the ink and eroded bone was much more deeply stained (Figs. 3 and 5 to 8 ).

All the above findings were tabulated according to which joint was involved; whether bone or cartilage was affected; the exact area affected and the severity of the damage. This information was combined with clinical information obtained before operation regarding the degrees of flexion and ulnar deviation of the proximal phalanx on the metacarpal head.

TABLE I

Age Distribution

\begin{tabular}{|c:c|}
\hline $\begin{array}{c}\text { Age } \\
\text { (years) }\end{array}$ & $\begin{array}{c}\text { Number of } \\
\text { patients }\end{array}$ \\
\hline $20-30$ & 2 \\
$30-40$ & 14 \\
$40-50$ & 15 \\
$50-60$ & 12 \\
$60-70$ & 2 \\
\hline Total . & 45 \\
\hline
\end{tabular}

TABLE II

DURATION OF HAND Symptoms

\begin{tabular}{|c|c|}
\hline $\begin{array}{c}\text { Duration } \\
\text { (years) }\end{array}$ & $\begin{array}{c}\text { Number of } \\
\text { patients }\end{array}$ \\
\hline 1 & 9 \\
$1-2$ & 10 \\
$2-3$ & 5 \\
$3-4$ & 6 \\
$4-5$ & 5 \\
$5-10$ & 7 \\
10 & 3 \\
\hline Total & 45 \\
\hline
\end{tabular}

TABLE III

Distribution of Metacarpo-PHALANGeAl JoINTS AND TyPe of OPERATION Performed

\begin{tabular}{|c|c|c|c|c|c|}
\hline \multirow{2}{*}{ Operation } & \multicolumn{5}{|c|}{ Metacarpo-phalangeal joints } \\
\hline & I & II & III & IV & V \\
\hline Synovectomy & 7 & 24 & 20 & 8 & 11 \\
\hline Arthroplasty & & 14 & 14 & 14 & 14 \\
\hline Total & 7 & 38 & 34 & 22 & 25 \\
\hline
\end{tabular}

\section{NORMAL ANATOMY OF THE METACARPO-PHALANGEAL JOINT}

A typical metacarpo-phalangeal joint is shown diagrammatically in Figure 1. The opposing bones are covered in hyaline cartilage. Because the surface area of the articular cartilage on the metacarpal head is greater than that on the base of the proximal phalanx, there is always an area of articular cartilage on the metacarpal head exposed, no matter what position the joint is placed in. In contrast the articular cartilage on the base of the proximal phalanx is always covered and in close contact with its opposing articular cartilage.

Anatomical studies (Kuczynski 1971) have shown that the normal synovial membrane which envelops the metacarpo-phalangeal joint conforms to a general plan. The synovium is attached around the margins of the articular cartilage but the synovial lining is reflected proximally along the metacarpal neck forming synovial pouches as shown in Figure 1. This synovial reflection is greatest on the dorsal surface of the metacarpal neck and to a much lesser extent on the radial and ulnar surfaces beneath the collateral ligaments. Because of the cartilaginous deep surface of the volar plate, only the most proximal part of the volar pouch is formed by a double layer of synovium (Backhouse 1969). The double layer of synovium 
forming these pouches lies directly on cortical bone with no intervening articular cartilage. Vascular foramina perforate the relatively thin cortical bone at these sites and carry nutrient vessels into the metacarpal head (Moberg 1969).

\section{PATHOLOGICAL FINDINGS}

Before operation all the metacarpo-phalangeal joints showed some degree of deformity. This varied from a mild flexion deformity in the early cases to extreme flexion with ulnar deviation and subluxation in some of the later cases.

The appearance of the joint at operation also varied from no abnormality of bone or cartilage in five joints to virtually complete destruction of the joint and fibrous ankylosis in seven joints. Between these two extremes there was a complete spectrum of bone and cartilage damage.

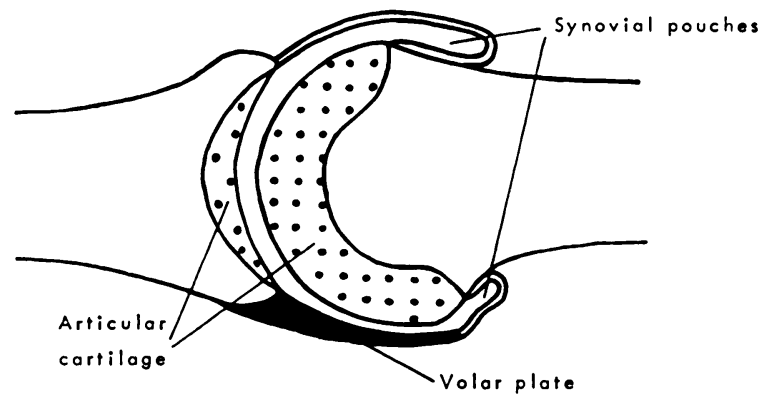

Fig. 1

Diagram of a sagittal section of a normal metacarpophalangeal joint. Note the large dorsal synovial pouch.

Those joints subjected to synovectomy all showed an active chronic synovitis with proliferation and vascularity of the synovium, whereas in many of those subjected to arthroplasty the synovium was much more fibrous and not so vascular. The most striking feature at synovectomy was the presence of a large synovial "slug" which lay on the neck of the metacarpal and represented the dorsal synovial pouch.

The findings within the joint can be divided into two main categories: 1) bone erosion. By this is meant a defect in the surface of the affected bone near the edge of the articular cartilage; 2) cartilage degeneration in which the articular cartilage is altered in some way but the underlying bone is unaffected.

As there were no significant pathological differences between the joints in right or left hands, the findings were grouped together. The metacarpo-phalangeal joints of the index and long fingers were the most often and usually the most severely affected in comparison with the other metacarpo-phalangeal joints, but the general pattern in all joints was the same.

Bone erosion-Metacarpal head-After removal of the large dorsal synovial "slug" a dorsal erosion covering a large area of the metacarpal neck was revealed in 81 per cent of cases (Fig. 2). In 60 per cent of cases this erosion was shallow but in the remaining 21 per cent it had extended through the cortical bone to a varying degree and the underlying cancellous bone was involved.

The second most common erosion occurred under either the ulnar or radial collateral ligaments; these two erosions occurred with nearly equal frequency. Seventy-six per cent had an erosion under the radial collateral ligament and 73 per cent under the ulnar collateral ligament. Some of these erosions were very deep and extended into the cancellous bone of the metacarpal head and in two cases they communicated with a large pseudocyst filled with granulation tissue.

VOL. 54 B, NO. 4, NOVEMBER 1972 
The presence of a volar erosion was often difficult to assess during synovectomy, but on the resected metacarpal heads this erosion when present tended to be relatively small, shallow and indistinct.

In 28 per cent of cases the dorsal, radial, ulnar and volar erosions had coalesced and formed a collar around the metacarpal neck with an island of articular cartilage in the centre. This island of articulating cartilage tended to become more and more localised to the volar and ulnar area of the metacarpal head as the disease progressed and the base of the proximal phalanx subluxed into flexion and ulnar deviation (Fig. 3).

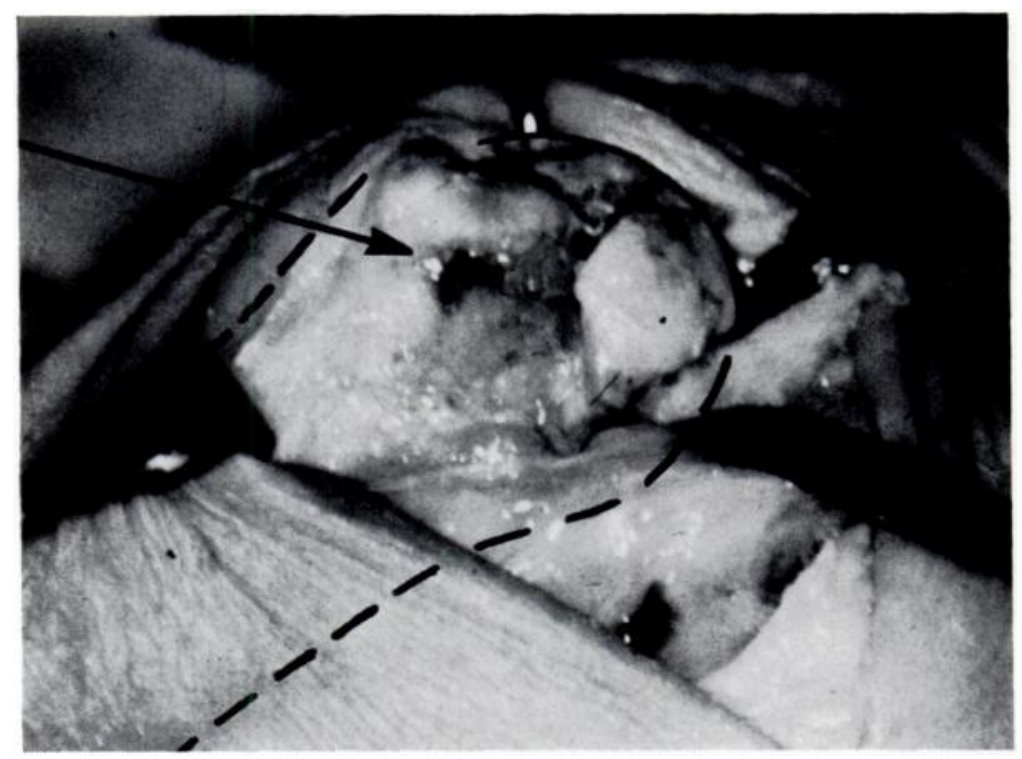

Fig. 2

A large dorsal erosion is shown with a furrow running forward on to the second metacarpal head. The outline of the metacarpal head and shaft have been sketched in for clarity.

In 8 per cent of cases the metacarpal head was virtually destroyed and in these cases there was usually extreme flexion, ulnar deviation and subluxation or dislocation of the joint. Base of proximal phalanx-Erosions were much less common here and not so extensive as on the head of the metacarpal. They occurred in 36 per cent of cases and involved only the margin at the base of the proximal phalanx in a ring-like manner (Fig. 4). The erosion was often greatest on the dorso-radial aspect and tended to undermine the articular cartilage which was preserved until very late in the disease.

In two cases the head of the metacarpal had been invaginated into the base of the proximal phalanx, both surfaces of the joint being destroyed. This type of situation has been described by Vaughan-Jackson (1969) as an "egg-cup erosion".

In only 8 per cent of cases was the articular cartilage on the base of proximal phalanx completely destroyed; this always coincided with complete destruction of the metacarpal head.

\section{DEGENERATION OF CARTILAGE}

Metacarpal head-Normal cartilage is smooth, white and shiny, with a translucent appearance. Any articular cartilage which did not conform to these criteria was considered abnormal. Degenerating cartilage was seen to pass through several stages. Initially it lost its sheen and became dull and soft. Later it became grey and fibrillated and later still slightly pitted. Eventually the underlying bone was exposed and an erosion developed. 


\section{DORSAL}

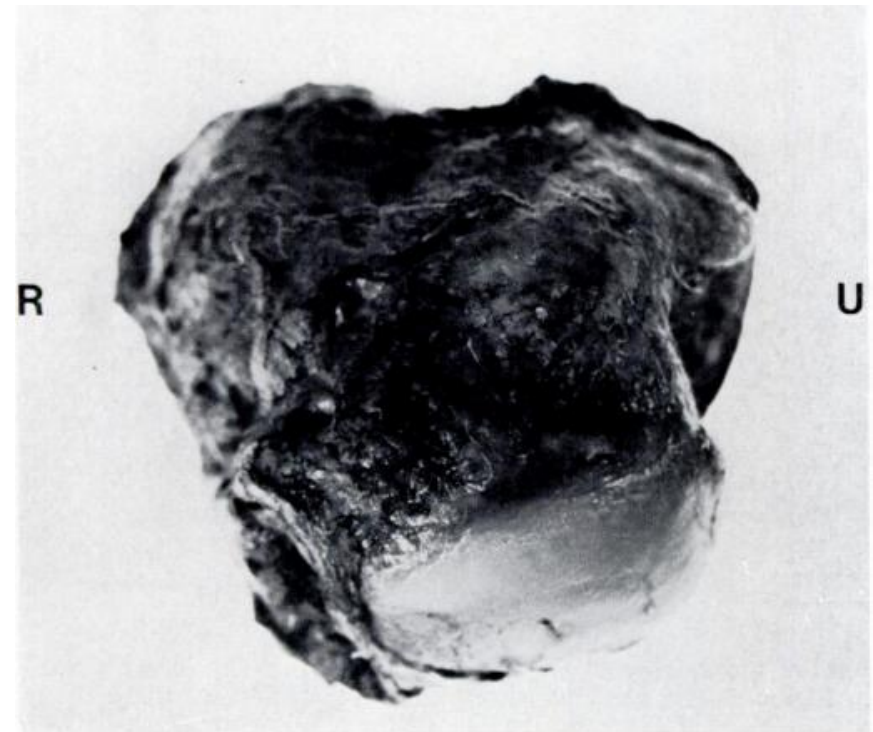

Fig. 3

Head-on view of the third left metacarpal head stained with indian ink. A collar of erosion is seen surrounding an island of articulating cartilage on the volar and ulnar aspect of the metacarpal head. The proximal phalanx was in marked flexion and ulnar deviation.

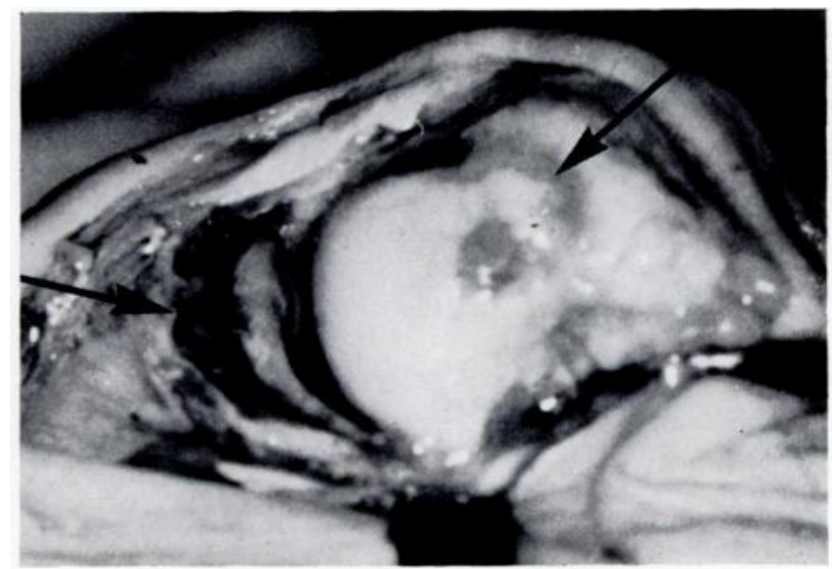

FIG. 4

A ring-like erosion is undermining the articular cartilage on the base of the proximal phalanx. Dorsal degeneration of cartilage is seen on the metacarpal head. 
There were three definite areas in which cartilage degeneration commenced. Two of these occurred with equal frequency and the third less commonly.

\section{DORSAL}

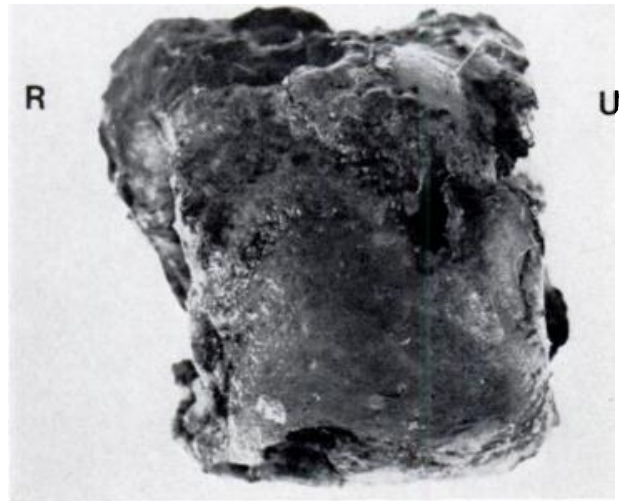

FIG. 5
DORSAL

$\mathbf{R}$

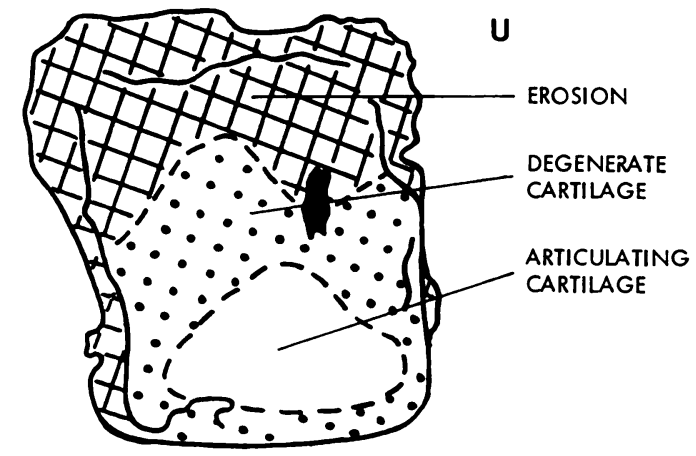

Fig. 6

Figure 5-Head-on view of the fourth left metacarpal head stained with indian ink. The proximal phalanx was in fixed flexion. Figure 6-Diagram indicating areas of erosion, cartilage degeneration and cartilage articulating with the base of the proximal phalanx.

\section{DORSAL}

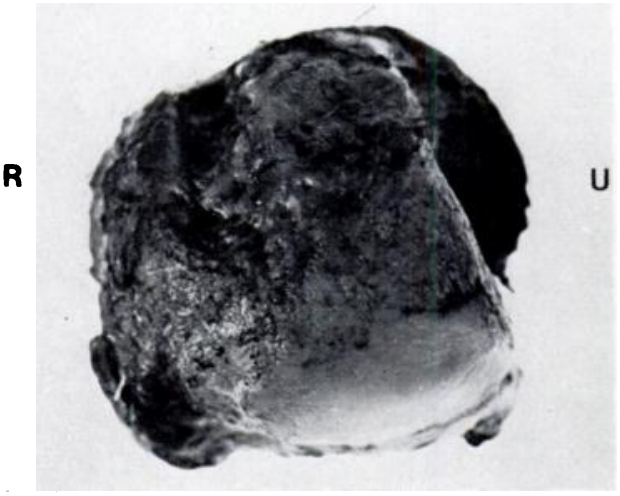

FIG. 7
DORSAL

$\mathbf{R}$

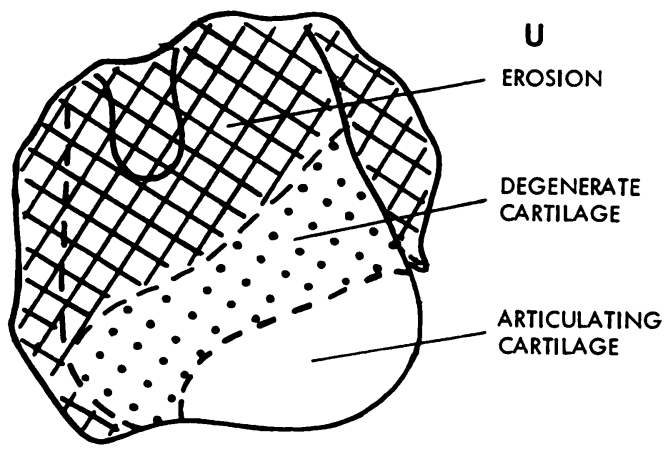

FIG. 8

Figure 7-Head-on view of second left metacarpal head stained with indian ink. The proximal phalanx was in fixed flexion and ulnar deviation. Figure 8-Diagram indicating areas of erosion, cartilage degeneration and cartilage articulating with the base of the proximal phalanx.

The two most common sites for degeneration were on the dorsal surface and the dorsoradial surface of the articulating cartilage on the metacarpal head. Degeneration was noted in either one or other of these areas in 66 per cent of cases.

Those joints which had only a flexion deformity as in the thumb had mainly dorsal degeneration (Figs. 4 to 6), whereas those joints in which ulnar deviation of the base of the proximal phalanx was added the degeneration mostly affected the dorso-radial aspect (Figs. 7 and 8 ). As the joint progressed in deformity so the area of degeneration crept down over 
the exposed area of the metacarpal head and was followed by the dorsal erosion which slowly extended into the degenerated area and often became deep and furrowed on the radial side (Figs. 7 and 8 ).

The least common type of degeneration occurred in the articulating cartilage over the most prominent part of the metacarpal head (Figs. 9 and 10), and affected 19 per cent of cases. This "isolated area" of degenerate cartilage lay in a sea of apparently normal-looking cartilage and usually appeared dull and purplish in colour. It was never seen to progress beyond this stage in this series of cases.

Base of proximal phalanx-Cartilage degeneration as described on the metacarpal head was seldom seen involving the articular cartilage on the base of a proximal phalanx which had not dislocated. Cartilage destruction when it did occur was due to the peripheral circular erosion slowly extending beneath the articular cartilage towards the centre of the joint.

Pathological findings related to the duration of symptoms-The earliest bone or cartilage changes seen within the joint were erosions at the margin of the articular cartilage on the metacarpal

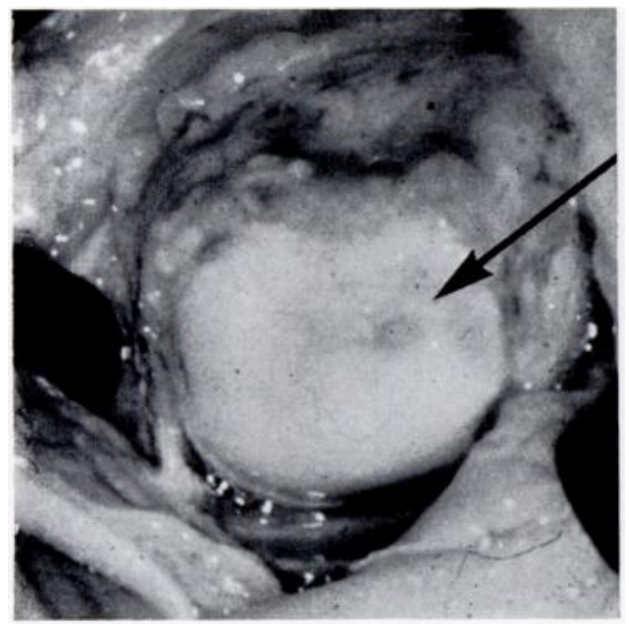

Fig. 9

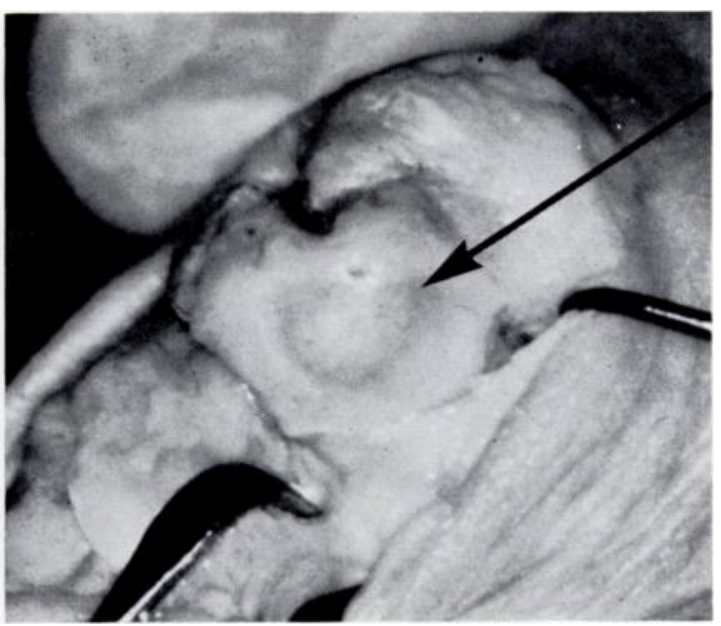

FIG. 10

"Isolated area" of cartilage degeneration on metacarpal head. Figure 9-Early stage on first metacarpal head. Figure 10-Later stage on second metacarpal head.

head at the sites already indicated. No one site of erosion constantly appeared any earlier than the others, although the erosions under the collateral ligaments ultimately became the deepest. With increasing duration of symptoms the degree of joint destruction became more severe although not all cases progressed at the same rate. A few cases showed quite extensive destructive changes within the first year of symptoms while others with a longer history were not so severely affected.

\section{A COMPARISON OF RADIOGRAPHIC AND PATHOLOGICAL FINDINGS}

The routine postero-anterior radiographs of the hands taken immediately before operation were assessed before each operation by the author and the findings were tabulated. These radiographic findings were now compared with the operative pathological findings (Fig. 11). From this one can see that the routine radiograph is a poor reflection of the actual pathological findings. Early bone erosion may be missed, early cartilage degeneration is never seen, and there may be a number of false positive results.

The most commonly seen erosion on the dorsum of the metacarpal head, present in 81 per cent of these cases, was only seen in 18 per cent of radiographs. The reason for this is that 
this erosion although large in area is relatively shallow and is viewed en face on routine radiography and therefore not easily seen.

Again the erosion under the ulnar collateral ligament was less easily seen on radiography than the erosion under the radial collateral ligament. The reason for this is probably that the hand placed on the radiographic plate is slightly rotated and the ulnar side of the metacarpal heads is slightly obscured. Also both of these erosions were much more extensive macroscopically than was indicated on the radiograph.

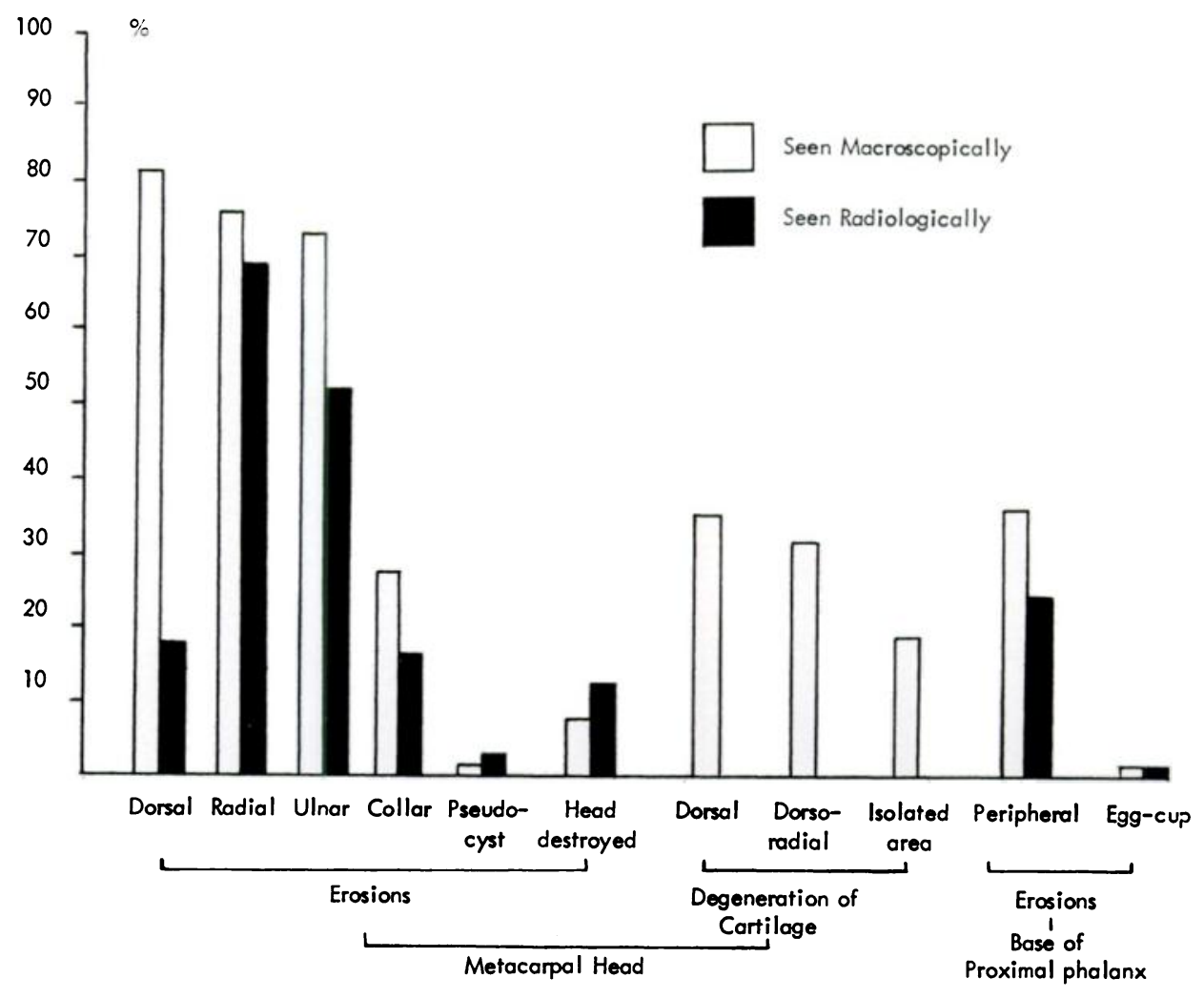

Fig. 11

Incidence of macroscopic findings compared with the radiological findings in 126 rheumatoid metacarpo-phalangeal joints.

The fact that the erosion at the base of the proximal phalanx tends to be circumferential is again not clearly indicated on routine radiography and appears only as localised erosions at either the radial or ulnar margins.

The degree of joint space narrowing as an index of cartilage damage becomes more difficult to assess as the deformity increases. The reason for this is that it is difficult to get a true antero-posterior radiographic projection of the deformed joint.

Both Norgaard (1965) and Brewerton (1967) have advocated using oblique or tangential projections for radiographs of the hands in an effort to visualise these hidden erosions of the metacarpo-phalangeal joints.

\section{DISCUSSION}

This study shows that both bone erosion and cartilage degeneration occur in certain specific sites and appear to progress in a systematic manner. I have shown that any study based on radiographic findings alone will not be truly representative of all the pathological changes within the joint. 
The large dorsal erosion, the erosions under the collateral ligaments and the volar erosion are all seen to develop in areas of cortical bone adjacent to articular cartilage which are covered by a double layer of synovial membrane forming the synovial pouches. The fact that the large dorsal erosion corresponds exactly with the large dorsal synovial pouch seems more than coincidental. Since the synovium is considered to be the initial site of pathological change in the rheumatoid joint, I suggest that this double layer of diseased tissue in contact with these specific areas of relatively thin cortical bone pierced by vascular foramina gives rise to the initial erosions. Moberg (1969) has suggested that the diseased synovium invades along these nutrient vessels, enlarging them further and initiating destruction. This may indeed be the mechanism by which synovial tissue enters the metacarpal head, causing pressure erosion of the cancellous bone and giving rise to pseudocyst formation.

As the joint becomes increasingly deformed by either flexion or by flexion and ulnar deviation of the proximal phalanx on the metacarpal head, so more of the articular cartilage on the dorsal or dorso-radial aspects of the metacarpal head becomes exposed. This uncovered

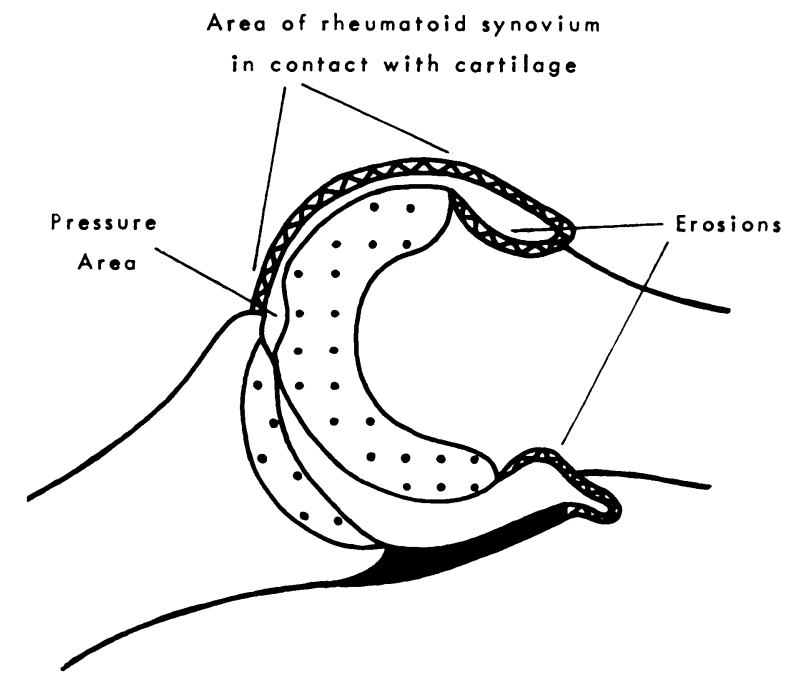

FIG. 12

Diagram of a sagittal section of a rheumatoid metacarpophalangeal joint with a flexion deformity and volar subluxation of the proximal phalanx on the metacarpal head.

articular cartilage now lies in continual contact with the diseased synovium (Fig. 12). Hamerman (1969) has stated that lysosomal enzymes released from proliferating rheumatoid synovium in direct contact with cartilage may gain access to the cartilage matrix and degrade it at a rate faster than the chondrocytes can replace it. Cartilage is eventually destroyed and the matrix is "replaced" by pannus. It is these areas of degenerating cartilage that we see on the dorsal and dorso-radial aspects of the metacarpal head (Figs. 4 to 8). Because the site of the degenerative cartilage is dependent on the area in contact with the diseased synovium it is therefore also dependent on the position of the deformed joint.

As the disease progresses so pannus originating at the articular edge of the large dorsal erosion extends into the already degraded area of articular cartilage and an actual erosion is formed. This erosion is an extension of the original dorsal erosion, and destruction is often most severe on the dorso-radial side where the head becomes furrowed (Figs. 7 and 8).

It is interesting to note that the "isolated area" of degenerate cartilage on the metacarpal head (Figs. 9 and 10) is not in contact with synovium and is surrounded by apparently normal articular cartilage. This "isolated area" lies under the dorsal lip of the proximal phalanx and 
one can only speculate on the way in which it is produced. It has been suggested that increased amounts of lysosomal enzymes in the joint synovial fluid may deplete the cartilage matrix (Hamerman 1969). This change is not visible macroscopically and is only revealed by a failure of the matrix to stain metachromatically. In the subluxed metacarpo-phalangeal joint the dorsal lip of the proximal phalanx will press on a localised area of the metacarpal head as the joint is extended (Fig. 12). If the matrix of the articular cartilage on this pressure area is already depleted an "isolated area" of macroscopically degenerate cartilage may be formed.

With progression of the disease and increasing deformity the remaining normal articular cartilage becomes more and more localised to the ulnar and volar aspects of the metacarpal head (Fig. 3), and lies under the "protection" of the base of the proximal phalanx. The rest of the metacarpal head is destroyed by the merging of the peripheral erosions.

As there are no synovial pouches associated with the base of the proximal phalanx, it is not surprising that the degree of erosion here is correspondingly less. Erosion occurs in a ring-like manner around the base of the phalanx where the synovium is attached to the margin of the articular cartilage (Fig. 4). This ring erosion is often greatest on the dorso-radial aspect of the base of the phalanx and adjacent to an "isolated area" of degenerate cartilage on the metacarpal head. I suggest that the increased size of the erosion at this site is also due to the mutual pressure of the dorso-radial lip of the base of the phalanx on the metacarpal head as the subluxed joint is extended (Fig. 12).

Degraded cartilage as seen on the head of the metacarpal is seldom seen on the base of a proximal phalanx which has not dislocated. The reason for this is that the articular cartilage is never exposed to direct contact with the synovium and is always in contact with its opposing articular cartilage on the head of the metacarpal. It is only in the very late stages when the head of the metacarpal is destroyed that the articular cartilage on the base of the proximal phalanx is also destroyed.

In conclusion, it can be said that although the pathological processes by which bone and cartilage are destroyed in rheumatoid arthritis are not yet fully understood, there are certain anatomical and mechanical factors which influence the actual sites of destruction and the progress of the disease. Bone erosion is shown to be initially related to the normal anatomy of the joint, and cartilage degeneration progresses in a systematic fashion which is predictable and related to the increasing deformity of the joint. The anatomical factors can be overcome by early removal of the diseased synovium from the predisposed areas although this result may not be permanent. The adverse mechanical factors may be diminished by encouraging joint movement and preventing deformity, thereby increasing the area of cartilage to cartilage contact and decreasing the area of cartilage in continual contact with the diseased synovium.

\section{SUMMARY}

1. One hundred and twenty-six metacarpo-phalangeal joints affected by rheumatoid arthritis were studied macroscopically at either synovectomy or arthroplasty.

2. The sites and extent of the initial erosion corresponded with the sites and size of the synovial pouches.

3. The areas of cartilage degeneration were related to the degree of flexion, ulnar deviation and subluxation of the proximal phalanx on the metacarpal head.

4. The routine radiographic findings were not a true reflection of all the pathological changes within the joint.

5. It is suggested that by encouraging joint movement and preventing deformity the degree of cartilage degeneration may be diminished.

I am grateful to Professor K. Vainio, Chief Surgeon, and Dr P. Raunio, Assistant Chief Surgeon, of the Rheumatism Foundation Hospital for permission to study their patients. 


\section{REFERENCES}

American Rheumatism Association (1959): Diagnostic Criteria for Rheumatoid Arthritis. 1958 Revision. Chairman Dr M. W. Ropes. Annals of the Rheumatic Diseases, 18, 49.

BACKhouse, K. M. (1969): Functional Asymmetry of the Hand Metacarpophalangeal Joints and its Relationship to Rheumatoid Deformities. An Anatomical Study. In Early Synovectomy in Rheumatoid Arthritis, p. 166. Edited by W. Hijmans, W. D. Paul and H. Herschel. Amsterdam: Excerpta Medica Foundation.

Brewerton, D. A. (1967): A Tangential Radiographic Projection for Demonstrating Involvement of Metacarpal Heads in Rheumatoid Arthritis. British Journal of Radiology, 40, 233.

Hamerman, D. (1969): Cartilage Changes in the Rheumatoid Joint. Clinical Orthopaedics and Related Research, 64, 91.

Hellum, C., and Vainio, K. (1968): Arthroplasty of the Metacarpophalangeal Joints in Rheumatoid Arthritis with Transposition of the Interosseous Muscles. Scandinavian Journal of Plastic and Reconstructive Surgery, 2, 139.

KUCZYNSKI, K. (1971): The Synovial Structures of the Normal and Rheumatoid Digital Joints. The Hand: Journal of the British Society for Surgery of the Hand, 3, 41.

Martel, W. (1964): The Pattern of Rheumatoid Arthritis in the Hand and Wrist. Radiologic Clinics of North America, 2, 221.

Martel, W., Hayes, J. T., and Duff, I. F. (1965): The Pattern of Bone Erosion in the Hand and Wrist in Rheumatoid Arthritis. Radiology, 84, 204.

Moberg, E. (1969): Cartilage Lesions. In Early Synovectomy in Rheumatoid Arthritis, p. 173. Edited by W. Hijmans, W. D. Paul and H. Herschel. Amsterdam: Excerpta Medica Foundation.

NorgaARD, F. (1965): Earliest Roentgenological Changes in Polyarthritis of the Rheumatoid Type: Rheumatoid Arthritis. Radiology, 85, 325.

Smith, R. J., and Kaplan, E. B. (1967): Rheumatoid Deformities at the Metacarpophalangeal Joints of the Fingers. Journal of Bone and Joint Surgery, 49-A, 31.

Vaughan-Jackson, O. J. (1969): Egg-cup Erosion. The Hand: Journal of the British Society for Surgery of the Hand, $1,9$. 